\title{
Infinite Existence Solutions of Fractional Systems with Lipschitz Nonlinearity
}

\author{
Rafik Guefaifia, ${ }^{1}$ Salah Mahmoud Boulaaras $\mathbb{D}^{2,3}$ Bahri Cherif, $^{2}$ and Taha Radwan $\mathbb{D}^{2,4}$ \\ ${ }^{1}$ Laboratory of Mathematics, Informatics and System (LAMIS), Larbi Tebessi University, Tebessa, Algeria \\ ${ }^{2}$ Department of Mathematics, College of Sciences and Arts, Qassim University, Ar Rass, Saudi Arabia \\ ${ }^{3}$ Laboratory of Fundamental and Applied Mathematics of Oran (LMFAO), University of Oran 1 Ahmed Ben Bella, Algeria \\ ${ }^{4}$ Department of Mathematics and Statistics, Faculty of Management Technology and Information Systems, Port Said University, \\ Port Said, Egypt \\ Correspondence should be addressed to Taha Radwan; t.radwan@qu.edu.sa
}

Received 2 November 2020; Revised 6 December 2020; Accepted 8 December 2020; Published 17 December 2020

Academic Editor: Maria Alessandra Ragusa

Copyright (C) 2020 Rafik Guefaifia et al. This is an open access article distributed under the Creative Commons Attribution License, which permits unrestricted use, distribution, and reproduction in any medium, provided the original work is properly cited.

The paper deals with the existence of infinitely many solutions of a class of perturbed nonlinear fractional $p$-Laplacian differential systems using one control parameter combined with the variational method.

\section{Introduction}

Fractional differential equations (FDEs) involve fractional derivatives of the form $\left(d^{\alpha} / d x^{\alpha}\right)(\alpha>0)$, where is not necessarily an integer. They are generalizations of the ordinary differential equations to a random (noninteger) order. FDEs have attracted considerable interest due to their ability to model complex phenomena in several fields of science, engineering, physics, biology, and economics (see [1-7]). In summary, many improvements have been made in the theory of partial calculus and partial differential equations and partial and ordinary differential equations (see [8-18], [2, 5]). Numerous studies have explored the existence and solutions of different nonlinear elementary and boundary value problems through the use of various nonlinear analysis tools and techniques (see, for example, [7, 19-38]). Some of these ways are the fixed point theorems, critical point theory, monotone iterative methods, coincidence degree theory, and variational methods (see [30]).
Motivated by the above, the interest of this paper is the infinite existence solutions of the following fractional system

$$
\begin{cases}{ }_{t} D_{T}^{\alpha}\left(\Phi_{p}\left({ }_{0} D_{t}^{\alpha} u(t)\right)\right)=\lambda F_{u}(t, u(t), v(t))+h_{1}\left(u_{1}\right), & \text { a.e. } t \in[0, T], \\ { }_{t} D_{T}^{\beta}\left(\Phi_{p}\left({ }_{0} D_{t}^{\beta} v(t)\right)\right)=\lambda F_{v}(t, u(t), v(t))+h_{2}\left(u_{2}\right), & \text { a.e. } t \in[0, T], \\ u(0)=u(T)=0, \quad v(0)=v(T)=0, & \end{cases}
$$

where $\lambda$ is a positive real parameter, $\alpha, \beta \epsilon(0 ; 1]$, and ${ }_{0} D_{t}^{\alpha}$, ${ }_{t} D_{T}^{\alpha}$ and ${ }_{0} D_{t}^{\beta},{ }_{t} D_{T}^{\beta}$ are the left and right Riemann-Liouville fractional derivatives of order $\alpha, \beta$, respectively, $\Phi_{p}(s)=$ $|s|^{p-2} s, p>1, \quad\left(H_{0}\right) F:[0, T] \times \mathbb{R}^{2} \rightarrow \mathbb{R}$, where $F(\cdot, u, v)$ is continuous in $[0, T]$ for any $(u, v) \in \mathbb{R}^{2}, F(t, \cdot, \cdot)$ is a $C^{1}$ function in $\mathbb{R}^{2}$, and $F_{s}$ is the partial derivative of $F$ with respect to $s$, and $h_{i}: \mathbb{R} \longrightarrow \mathbb{R}$ are two Lipschitz continuous 
functions of order $(p-1)$ with Lipschitzian constants $L_{i}>0$ for $1 \leq i \leq 2$, i.e.,

$$
\left|h_{i}\left(x_{1}\right)-h_{i}\left(x_{2}\right)\right| \leq L_{i}\left|x_{1}-x_{2}\right|^{p-1} \text {. }
$$

\section{Preliminaries}

We give some basic lemmas and notations and construct a variational framework in order to apply critical point theory to prove the existence of an infinite number of solutions to the system (1).

Let $X$ be a real Banach space, and in addition, let $Y_{X}$ denote the class of all functionals

$$
\phi=X \longrightarrow \mathbb{R},
$$

that possess the following property:

If $\left\{w_{n}\right\}$ is a sequence in $X$ converge weakly to $w \in X$ with $\lim _{n \rightarrow \infty} \inf \phi\left(w_{n}\right) \leq \phi(w)$; thus, $\left\{w_{n}\right\}$ has a subsequence converge strongly to $w$.

For offer, if $X$ is uniformly convex and $S:[0,+\infty) \longrightarrow \mathbb{R}$ is a continuous strictly increasing function, then the functional $w \longrightarrow S(\|w\|)$ belongs to $Y_{X}$.

Definition 1 (see Kilbas et al. [4] chapter 2, p. 87). Let $u$ be a function defined on $[a, b]$. The right and left RiemannLiouville fractional derivatives of order $>0$ for a function $u$ are defined by

$$
{ }_{a} D_{t}^{\alpha} u(t):=\frac{d^{n}}{d t^{n}} D_{t}^{\alpha-n} u(t)=\frac{1}{\Gamma(n-\alpha)} \frac{d^{n}}{d t^{n}} \int_{a}^{t}(t-s)^{n-\alpha-1} u(s) d s,
$$

$$
{ }_{a} D_{b}^{\alpha} u(t):=(-1)^{n} \frac{d^{n}}{d t^{n}} D_{b}^{\alpha-n} u(t)=\frac{(-1)^{n}}{\Gamma(n-\alpha)} \frac{d^{n}}{d t^{n}} \int_{a}^{b}(t-s)^{n-\alpha-1} u(s) d s,
$$

for all $t \in[a, b]$, provided the right-hand sides are pointwise defined on $[a, b]$, where $n-1 \leq \alpha<n$ and $n \in \mathbb{N}$.

Here, $\Gamma(\alpha)$ is the standard gamma function given by

$$
\Gamma(\alpha):=\int_{0}^{+\infty} \varkappa^{\alpha-1} e^{-\varkappa} d \varkappa .
$$

Set $A C^{n}([a, b], \mathbb{R})$ the functions space $u:[a, b] \longrightarrow \mathbb{R}$ such that

$$
u \in C^{n-1}([a, b], \mathbb{R}),
$$

with

$$
u^{(n-1)} \in A C^{n}([a, b], \mathbb{R}) .
$$

As usual, $C^{n-1}([a, b], \mathbb{R})$ denotes the mapping set having $(n-1)$ times continuously differentiable on $[a, b]$. In particularly, we have

$$
A C([\mathrm{a}, \mathrm{b}], \mathbb{R}):=A C^{1}([\mathrm{a}, \mathrm{b}], \mathbb{R}) .
$$

Definition 2 (see [31]). Let $0<\alpha \leq 1$, for $1<p<\infty$ the derivative fractional space

$E_{\alpha}^{p}=\left\{u(t) \in L^{p}([0, T], \mathbb{R}){ }_{0} D_{t}^{\alpha} u(t) \in L^{p}([0, T], \mathbb{R}), u(0)=u(T)=0\right\}$.

Thus, for all $u \in E_{\alpha}^{p}$, we de ne the norm for $E_{\alpha}^{p}$ as follows:

$$
\|u\|_{\alpha}=\left(\int_{0}^{T}|u(t)|^{p} d t+\int_{0}^{T}\left|{ }_{0} D_{t}^{\alpha} u(t)\right|^{p} d t\right)^{1 / p} .
$$

Lemma 3 (see [3]). Let $0<\alpha \leq 1$ and $1<p<\infty$. For any $u$ $\in E_{\alpha}^{p}$, we have

$$
\|u\|_{L^{p}} \leq \frac{T^{\alpha}}{\Gamma(\alpha+1)}\left\|_{0} D_{t}^{\alpha} u\right\|_{L^{p}}
$$

Also, if $\alpha>p$ and $1 / p+1 / q=1$, then

$$
\|u\|_{\infty} \leq \frac{T^{\alpha-1 / p}}{\Gamma(\alpha)((\alpha-1) q+1)^{1 / q}}\left\|_{0} D_{t}^{\alpha} u\right\|_{L^{p}}
$$

Under the result of Lemma 3, we note that

$$
\|u\|_{L^{p}} \leq \frac{T^{\alpha-1 / p}}{\Gamma(\alpha+1)}\left\|_{0} D_{t}^{\alpha} u\right\|_{L^{p}}
$$

for $0<\alpha \leq 1$, and

$$
\|u\|_{\infty} \leq \frac{T^{\alpha-1 / p}}{\Gamma(\alpha)((\alpha-1) q+1)^{1 / q}}\left\|_{0} D_{t}^{\alpha} u\right\|_{L^{p}}
$$

for $\alpha>p$ and $1 / p+1 / q=1$.

Under (14), we can see that (11) is equivalent to the following norm:

$$
\|u\|_{\alpha}=\left(\left.\left.\int_{0}^{T}\right|_{0} D_{t}^{\alpha} u(t)\right|^{p} d t\right)^{1 / p}, \quad \forall u \in E_{\alpha}^{p}
$$

For $0<\beta \leq 1,1<p<\infty$. Analogous to the space $E_{\alpha}^{p}$, we define the fractional derivative space $E_{\alpha}^{p}$ as

$$
\left\{v(t) \in L^{p}([0, T], \mathbb{R})_{0} D_{t}^{\beta} v(t) \in L^{p}([0, T], \mathbb{R}), v(0)=v(T)=0\right\} .
$$

Then, for any $v \in E_{\beta}^{p}$, the norm of $E_{\beta}^{p}$ is defined by

$$
\|v\|_{\beta}=\left(\int_{0}^{T}|v(t)|^{p} d t+\left.\int_{0}^{T}{ }_{0} D_{t}^{\beta} v(t)\right|^{p} d t\right)^{1 / p}, \quad \forall v \in E_{\beta}^{p} .
$$


Similar with (14) and (15), we get

$$
\|v\|_{L^{p}} \leq \frac{T^{\beta}}{\Gamma(\beta+1)}\left\|_{0} D_{t}^{\alpha} v\right\|_{L^{p}}
$$

for $0<\beta \leq 1$, and

$$
\|v\|_{\infty} \leq \frac{T^{\beta-1 / p}}{\Gamma(\beta)((\beta-1) q+1)^{1 / q}}\left\|_{0} D_{t}^{\alpha} v\right\|_{L^{p}} .
$$

Moreover, if $0<\beta \leq 1$ and $1 / p+1 / q=1$, then, based upon (19), the weighted norm

$$
\|v\|_{\beta}=\left(\int_{0}^{T}\left|{ }_{0} D_{t}^{\beta} v(t)\right|^{p} d t\right)^{1 / p}
$$

is equivalent to (18), for every $v \in E_{\beta}^{p}$.

In the following discussion, for any $u \in E_{\alpha}^{p}, v \in E_{\beta}^{p}$ denote the space of $X=E_{\alpha}^{p} \times E_{\beta}^{p}$ with the norm

$$
\|(u, v)\|_{X}=\left(\|u\|_{\alpha}^{p}+\|v\|_{\beta}^{p}\right)^{1 / p}, \quad \forall(u, v) \in X
$$

where $\|u\|_{\alpha}$ and $\|u\|_{\beta}$ are defined in (16) and (21), respectively.

Clearly, $X$ is embedded compactly on

$$
C^{0}([0, T], \mathbb{R}) \times C^{0}([0, T], \mathbb{R}) .
$$

Lemma 4 (see [33]). For $0<\alpha, \beta \leq 1$ and $1<p<\infty$. The derivative fractional space $X$ is a reflexive separable Banach space.

Lemma 5. Assume that $1 / p<\alpha \leq 1$ and the sequence $\left\{u_{n}\right\}$ converge weakly to $u$ in $E_{\alpha}^{p}$, i.e., $u_{n} \rightarrow u$. Then, $\left\{u_{n}\right\}$ converges strongly to $u$ in $C([0, T], \mathbb{R})$, i.e., $\left\|u_{n}-u\right\|_{\infty} \longrightarrow 0$, as $n \longrightarrow$ $+\infty$.

Definition 6 (see [3]). We point out to a weak solution to the system (1), for all $(u, v) \in X$ such that

$$
\begin{aligned}
& \int_{0}^{T} \Phi_{p}\left({ }_{0} D_{t}^{\alpha} u(t)\right)_{0} D_{t}^{\alpha} x(t) d t+\int_{0}^{T} \Phi_{p}\left({ }_{0} D_{t}^{\alpha} v(t)\right)_{0} D_{t}^{\beta} y(t) d t \\
& \quad-\int_{0}^{T} h_{1}(u(t)) x(t) d t-\int_{0}^{T} h_{2}(v(t)) y(t) d t \\
& \quad-\lambda \int_{0}^{T}\left(F_{u}(t, u(t), v(t)) x(t)+F_{v}(t, u(t), v(t)) y(t)\right) d t=0,
\end{aligned}
$$

for all $(x, y) \in X$.
We define for all $x \in \mathbb{R}$ :

$$
H_{i}(x)=\int_{0}^{x} h_{i}(z) d z, \Theta_{i}(x)=\int_{0}^{T} H_{i}(x(s)) d s \text { for all } i=1,2,
$$

for every $t \in[0, T]$.

Lemma 7. Let $h_{1}, h_{2}: \mathbb{R} \rightarrow \mathbb{R}$ satisfy (2) and $H_{i}(x), \Theta_{i}(x)$, $i=1,2$, defined by (25). Thus, $\Theta(u, v): X \longrightarrow \mathbb{R}$ defined by

$$
\Theta(u, v)=\Theta_{1}(u)+\Theta_{2}(v)=\int_{0}^{T} H_{1}(u(t)) d t+\int_{0}^{T} H_{2}(v(t)) d t
$$

is a Gâteaux function weakly sequentially differentiable over $X$ with

$\Theta^{\prime}(u, v)(x, y)=\int_{0}^{T} h_{1}(u(t)) x(t) d t+\int_{0}^{T} h_{2}(v(t)) y(t) d t, \quad$ for all $(x, y) \in X$.

Proof. Assume that

$$
\left\{\left(u_{n}, v_{n}\right)\right\} \subset X,\left(u_{n}, v_{n}\right) \rightarrow(u, v) \text { in } X,
$$

as $n \rightarrow+\infty$. According to Lemma 5 that $\left(u_{n}, v_{n}\right)$ converges uniformly to $(u, v)$ on $[0, T]$. Then, there exists $c_{1}, c_{2}>0$ such that $\left\|u_{n}\right\|_{\infty} \leq c_{1}$ and $\left\|v_{n}\right\|_{\infty} \leq c_{2}$ for any $n \in \mathbb{N}$.

Then,

$$
\begin{aligned}
& \left|H_{1}\left(u_{n}(t)\right)-H_{1}(u(t))\right| \leq\left. L_{1}\left|\int_{u(t)}^{u_{n}(t)}\right| s\right|^{p-1} d s \mid \\
& \leq \frac{L_{1}}{p}\left(\left|u_{n}(t)\right|^{p}+|u(t)|^{p}\right) \leq \frac{L_{1}}{p}\left(c_{1}^{p}+\|\left. u(t)\right|_{\infty} ^{p}\right), \\
& \left|H_{2}\left(v_{n}(t)\right)-H_{2}(v(t))\right| \leq\left. L_{2}\left|\int_{v(t)}^{v_{n}(t)}\right| s\right|^{p-1} d s \mid \\
& \leq \frac{L_{2}}{p}\left(\left|v_{n}(t)\right|^{p}+|v(t)|^{p}\right) \leq \frac{L_{2}}{p}\left(c_{2}^{p}+\|v(t)\|_{\infty}^{p}\right),
\end{aligned}
$$

for any $n \in \mathbb{N}$ and $t \in[0, T]$. Furthermore, $H_{1}\left(u_{n}(t)\right) \longrightarrow$ $H_{1}(u(t))$ and $H_{2}\left(v_{n}(t)\right) \longrightarrow H_{2}(v(t))$ at every $t \in[0, T]$, and by the Lebesgue Convergence Theorem

$$
\begin{aligned}
\Theta\left(u_{n}, v_{n}\right)= & \int_{0}^{T} H_{1}\left(u_{n}(t)\right) d t+\int_{0}^{T} H_{2}\left(v_{n}(t)\right) d t \longrightarrow \int_{0}^{T} H_{1}(u(t)) d t \\
& +\int_{0}^{T} H_{2}(v(t)) d t=\Theta(u, v) .
\end{aligned}
$$

Now we prove the Gâteaux differentiability of $\Theta$. Assume 
that $u, x \in E_{\alpha}^{p}$ and $s \neq 06$; thus,

$$
\begin{aligned}
& \left|\frac{\Theta_{1}(u+s x)-\Theta_{1}(u)}{s}-\int_{0}^{T} h_{1}(u(t)) x(t) d t\right| \\
& \quad \leq \int_{0}^{T}\left|\frac{H_{1}(u+s x)-H_{1}(u)}{s}-h_{1}(u(t)) x(t)\right| d t \\
& \quad=\int_{0}^{T}\left|h_{1}(u(t))+s \zeta(t) x(t)-h_{1}(u(t))\right||x(t)| d t \\
& \quad \leq L_{1}\|x\|_{\infty}^{p}|s|,
\end{aligned}
$$

where

$$
0<\zeta(t)<1, \quad t \in[0, T]
$$

Thus

$$
\Theta_{1}: E_{\alpha}^{p} \longrightarrow \mathbb{R}
$$

is a Gâteaux differentiable for all $u \in E_{\alpha}^{p}$.

Likewise, we have

$$
\Theta_{2}: E_{\beta}^{p} \longrightarrow \mathbb{R},
$$

which is a Gâteaux differentiable for all $v \in E_{\alpha}^{p}$.

Therefore,

$$
\Theta: X \longrightarrow \mathbb{R}
$$

is a Gâteaux differentiable for all $(u, v) \in X$ with its derivative

$$
\Theta^{\prime}(u, v)(x, y)==\int_{0}^{T} h_{1}(u(t)) x(t) d t+\int_{0}^{T} h_{2}(v(t)) y(t) d t,(x, y) \in X .
$$

For any three elements $\left(u_{1}, v_{1}\right),\left(u_{2}, v_{2}\right)$, and $(x, y)$ of $X$, it is easy to see that

$$
\begin{aligned}
& \left(\Theta^{\prime}\left(u_{1}, v_{1}\right)-\Theta^{\prime}\left(u_{2}, v_{2}\right)\right)(x, y)=\int_{0}^{T}\left(h_{1}\left(u_{1}\right)-h_{1}\left(u_{2}\right) x(t)\right) d t \\
& \quad+\int_{0}^{T}\left(h_{2}\left(v_{1}\right)-h_{2}\left(v_{2}\right) y(t)\right) d t \leq L_{1} \int_{0}^{T}\left|u_{1}-u_{2}\right|^{p-1}|x(t)| d t \\
& \quad+L_{2} \int_{0}^{T}\left|v_{1}-v_{2}\right|^{p-1}|y(t)| d t \leq \frac{L_{1} T^{\alpha-1 / p}}{\Gamma(\alpha)((\alpha-1) q+1)^{1 / p}} \\
& \quad \cdot\left\|u_{1}-u_{2}\right\|_{\infty}^{p-1}\|x\|_{\alpha}+\frac{L_{2} T^{\beta-1 / p}}{\Gamma(\beta)((\beta-1) q+1)^{1 / p}} \\
& \quad \cdot\left\|v_{1}-v_{2}\right\|_{\infty}^{p-1}\|y\|_{\alpha},
\end{aligned}
$$

which implies

$$
\left\|\Theta^{\prime}\left(u_{1}, v_{1}\right)-\Theta^{\prime}\left(u_{2}, v_{2}\right)\right\|_{X} \leq T^{*}\left(\left\|u_{1}-u_{2}\right\|_{\infty}^{p-1}+\left\|v_{1}-v_{2}\right\|_{\infty}^{p-1}\right),
$$

where

$$
T^{*}:=\max \left\{\frac{L_{1} T^{\alpha-1 / p}}{\Gamma(\alpha)((\alpha-1) q+1)^{1 / q}}, \frac{L_{2} T^{\beta-1 / p}}{\Gamma(\beta)((\beta-1) q+1)^{1 / q}}\right\} .
$$

Hence, $\Theta^{\prime}: X \longrightarrow X^{*}$ is a compact operator.

Similarly to the proof of Theorem 5.1 of [4], we have

Lemma 8 (see [36]). Let $1 / p<\alpha, \beta \leq 1$, and $(u, v) \in X$. If $(u, v)$ is a nontrivial weak solution of problem (1), then $(u, v)$ is also a nontrivial solution of problem (1).

Our analysis is mainly based on the following critical points theorem of Bonanno and Molica Bisci [36], which is a more precise result of Ricceri ([37], Theorem 2.5).

Lemma 9 (see [[36], Theorem 2.1]). Let $X$ be a reflexive real Banach space. Let $\phi, \Psi: X \longrightarrow \mathbb{R}$ be two Gâteaux differentiable functionals such that $\phi$ is sequentially weakly lower semicontinuous, strongly continuous, and coercive and $\Psi$ is sequentially weakly upper semicontinuous. For every $r>\inf _{X} \phi$, put

$$
\begin{gathered}
\varphi(r)=\inf _{\left.\left.u \in \phi^{-1}(]-\infty, r\right]\right)} \frac{\sup _{\left.\left.v \in \phi^{-1}(]-\infty, r\right]\right)} \Psi(v)-\Psi(u)}{r-\phi(u)}, \\
\gamma=: \lim _{r \rightarrow+\infty} \inf \varphi(r), \delta=: \lim _{r \rightarrow\left(\inf _{X} \phi\right)^{+}} \inf \varphi(r) .
\end{gathered}
$$

Then,

(1) If $\gamma<+\infty$ and $\lambda \in] 0,1 / \gamma[$, the following alternative holds: either the functional $\phi-\lambda \Psi$ has a global minimum or there exists a sequence $\left\{u_{n}\right\}$ of local minima $\phi-\lambda \Psi$ such that $\lim _{n \rightarrow+\infty} \phi\left(u_{n}\right)=+\infty$

(2) If $\gamma<+\infty$ and $\lambda \in] 0,1 / \delta[$, the following alternative holds: either there exists a global minimum of $\phi$ or the following alternative holds: either there exists a global minimum of $\phi-\lambda \Psi$ or there exists a sequence $\left\{u_{n}\right\}$ of pairwise distinct local minima of $\phi-\lambda \Psi$, with $\lim _{n \rightarrow+1} \phi\left(u_{n}\right)=\inf _{X} \phi$, which weakly converges to a global minimum of $\phi$

\section{Main Results}

Here, we prove our main results.

Setting

$$
k:=\min \left\{1-\frac{L_{1} T^{p \alpha}}{(\Gamma(\alpha+1))^{p}}, 1-\frac{L_{2} T^{p \beta}}{(\Gamma(\beta+1))^{p}}\right\}
$$

$\rho:=\max \left\{1+\frac{L_{1} T^{p \alpha}}{(\Gamma(\alpha+1))^{p}}, 1+\frac{L_{2} T^{p \beta}}{(\Gamma(\beta+1))^{p}}\right\}$, 


$$
M=\max \left\{\frac{T^{p \alpha-1}}{(\Gamma(\alpha))^{p}((\alpha-1) q+1)^{p / q}}, \frac{T^{p \beta-1}}{(\Gamma(\beta))^{p}((\beta-1) q+1)^{p / q}}\right\} .
$$

For a given constant $\theta \in(1 / p, 0)$, set

$$
\begin{aligned}
P(\alpha, \theta)= & \frac{1}{p(\theta T)^{p}}\left\{\int_{0}^{\theta T} t^{p(1-\alpha)} d t+\int_{\theta T}^{(1-\theta) T}\left(t^{1-\alpha}-(t-\theta T)^{1-\alpha}\right)^{p} d t\right. \\
& \left.+\int_{(1-\theta) T}^{T}\left[\left(t^{1-\alpha}-(t-\theta T)^{1-\alpha}\right)-(t-((1-\theta) T))^{1-\alpha}\right]^{p}\right\},
\end{aligned}
$$

$$
\begin{aligned}
Q(\beta, \theta)= & \frac{1}{p(\theta T)^{p}}\left\{\int_{0}^{\theta T} t^{p(1-\beta)} d t+\int_{\theta T}^{(1-\theta) T}\left(t^{1-\beta}-(t-\theta T)^{1-\beta}\right)^{p} d t\right. \\
& \left.+\int_{(1-\theta) T}^{T}\left[\left(t^{1-\beta}-(t-\theta T)^{1-\beta}\right)-(t-((1-\theta) T))^{1-\beta}\right]^{p}\right\} .
\end{aligned}
$$

For any $d>0$, we denote by $\Omega(d)$ the set

$$
\left\{(x, y) \in \mathbb{R}^{2}: \frac{1}{p}|x|^{p}+\frac{1}{p}|y|^{p} \leq d\right\} .
$$

Theorem 10. Suppose that $k>0$ and (HO) hold. In addition,

(H1) $1 / p \leq \alpha, \beta<1$

(H2) $F(t, x, y) \geq 0$ for any $(t, x, y)[0, T] \in[0,+\infty)[0,+\infty)$

(H3) there exists $\theta \in(0,1 / p)$ where if we set

$$
\begin{aligned}
& A_{\infty}=\lim _{\xi \rightarrow+\infty} \inf \frac{\int_{0}^{T} \sup _{|x|+|y| \leq \xi} F(t, x, y) d t}{\xi^{p}}, \\
& B_{\infty}=\lim _{\xi \rightarrow+\infty} \sup \frac{\int_{\theta T}^{(1-\theta) T} F(t, \Gamma(2-\alpha) \xi, \Gamma(2-\beta) \xi) d t}{\xi^{p}},
\end{aligned}
$$

one has

$$
A_{\infty}<\frac{k}{2 p M \rho \Delta} B_{\infty}
$$

where

$$
\Delta=\max \{P(\alpha, \theta), Q(\beta, \theta)\},
$$

and $M$ is given in (45).

Then, for every

$$
\lambda \in] \frac{\rho \Delta}{B_{\infty}}, \frac{k}{2 p M A_{\infty}}[.
$$

(1) has an unbounded sequence in X (weak solutions).
Proof. Our goal is to apply a portion (1) of Lemma 9 to problem (1). First, by taking

$$
X=E_{\alpha}^{p} \times E_{\beta}^{p},
$$

endowed with $\|(u, v)\|_{X}$ similar to what is considered in (22). We define the following functional

$$
I_{\lambda}(u, v)=\phi(u, v)-\lambda \Psi(u, v),
$$

for all $(u, v) \in X$, where

$$
\begin{aligned}
& \phi(u, v)=\frac{1}{p}\|u\|_{\alpha}^{p}+\frac{1}{p}\|v\|_{\beta}^{p}-\Theta(u, v), \\
& \Psi(u, v)=\int_{0}^{T} F(t, u(t), v(t)) d t .
\end{aligned}
$$

Since $X$ is embedded compact in

$$
C^{0}([0, T], \mathbb{R}) \times C^{0}([0, T], \mathbb{R}),
$$

it is well known that is a well-defined Gâteaux differentiable functional whose Gâteaux derivative at the point $(u, v) \in X$ is the functional $\Psi^{\prime}(u, v) \in X *$, given by

$$
\begin{aligned}
\Psi^{\prime}(u, v)(x, y)= & \int_{0}^{T}\left(F_{u}(t, u(t), v(t))\right) x(t) d t \\
& +\int_{0}^{T}\left(F_{v}(t, u(t), v(t))\right) y(t) d t,
\end{aligned}
$$

for every $(x, y) \in X$.

We claim that the functional $\Psi$ is a sequentially weakly upper semicontinuous functional on $X$. Indeed, for fixed $(u$, $v) \in X$, suppose that $\left\{\left(u_{n}, v_{n}\right)\right\} \subset X,\left(u_{n}, v_{n}\right) \rightarrow(u, v)$ in $X$ as $n \longrightarrow+\infty$. Then, $\left(u_{n}, v_{n}\right)$ converges uniformly to $(u, v)$ on $[0, T]$. Hence,

$$
\begin{gathered}
\lim _{n \rightarrow+\infty} \sup \Psi\left(u_{n}, v_{n}\right) \leq \int_{0}^{T} \lim _{n \rightarrow+\infty} \sup F\left(t, u_{n}(t), v_{n}(t)\right) d t \\
\quad=\int_{0}^{T} F(t, u,(t), v(t)) d t=\Psi(u, v),
\end{gathered}
$$

which implies that it is sequentially weakly upper semicontinuous. Hence, the claim is true.

Concerning the functional $\phi$, we can show that what is defined by (56) is a sequentially weakly lower semicontinuous, strongly continuous, and coercive functional on $X$. In fact since (2) holds for every $x_{1}, x_{2} \in \mathrm{R}$ and $h_{1}(0)=h_{2}(0)=0$, one has $\left|h_{i}(x)\right| \leq L_{i}|x|^{p-1}, i=1,2$, for all $x \in \mathbb{R}$. It follows from 
(14), (20), and Lemma 5 that

$$
\begin{gathered}
\phi(u, v) \geq \frac{1}{p}\|u\|_{\alpha}^{p}+\frac{1}{p}\|v\|_{\beta}^{p}-\left|\int_{0}^{T} H_{1}(u(t)) d t\right|-\left|\int_{0}^{T} H_{2}(v(t)) d t\right| \\
\geq \frac{1}{p}\|u\|_{\alpha}^{p}+\frac{1}{p}\|v\|_{\beta}^{p}-\frac{L_{1}}{p} \int_{0}^{T}|u(t)|^{p} d t-\frac{L_{2}}{p} \int_{0}^{T}|v(t)|^{p} d t \\
\geq\left(\frac{1}{p}-\frac{L_{1} T^{p \alpha}}{p(\Gamma(\alpha+1))^{p}}\right)\|u\|_{\alpha}^{p}+\left(\frac{1}{p}-\frac{L_{2} T^{p \beta}}{p(\Gamma(\beta+1))^{p}}\right)\|v\|_{\beta}^{p},
\end{gathered}
$$

for all $(u, v) \in X$ and similarly

$$
\begin{aligned}
\phi(u, v) & \leq \frac{1}{p}\|u\|_{\alpha}^{p}+\frac{1}{p}\|v\|_{\beta}^{p}+\left|\int_{0}^{T} H_{1}(u(t)) d t\right|+\left|\int_{0}^{T} H_{2}(v(t)) d t\right| \\
& \leq\left(\frac{1}{p}-\frac{L_{1} T^{p \alpha}}{p(\Gamma(\alpha+1))^{p}}\right)\|u\|_{\alpha}^{p}+\left(\frac{1}{p}-\frac{L_{2} T^{p \beta}}{p(\Gamma(\beta+1))^{p}}\right)\|v\|_{\beta}^{p} \\
& \leq \frac{\rho}{p}\left(\|u\|_{\alpha}^{p}+\|v\|_{\beta}^{p}\right)
\end{aligned}
$$

for all $(u, v) \in X$. So $\phi$ is coercive.

Moreover, $\phi+\Theta$ is a continuous functional on $X$, and $\Theta$, from Lemma 5, is Gâteaux differentiable sequentially weakly continuous and therefore continuous on $X$, then $\phi$ is a continuous functional on $X$. It is not difficult to verify that the functional is a Gâteaux differentiable functional with the differential

$$
\begin{aligned}
\phi^{\prime}(u, v)(x, y)= & \int_{0}^{T} \Phi_{p}\left({ }_{0} D_{t}^{\alpha} u(t)\right)_{0} D_{t}^{\alpha} x(t) d t \\
& +\int_{0}^{T} \Phi_{p}\left({ }_{0} D_{t}^{\alpha} v(t)\right)_{0} D_{t}^{\beta} y(t) d t \\
& -\int_{0}^{T} h_{1}(u(t)) x(t) d t-\int_{0}^{T} h_{2}(v(t)) y(t) d t .
\end{aligned}
$$

Furthermore, $\phi$ is also sequentially weakly lower semicontinuous on $X$ since $\Theta$ is sequentially weakly lower semicontinuous, and if $\left(u_{n}, v_{n}\right) \rightarrow(u, v)$ in $X$ then

$$
\begin{aligned}
& \lim _{n \rightarrow+\infty} \inf \phi\left(u_{n}, v_{n}\right)=\lim _{n \rightarrow+\infty} \inf \left(\frac{1}{p}\|u\|_{\alpha}^{p}+\frac{1}{p}\|v\|_{\beta}^{p}\right) \\
& -\lim _{n \rightarrow+\infty} \Theta\left(u_{n}, v_{n}\right) \geq \frac{1}{p}\|u\|_{\alpha}^{p}+\frac{1}{p}\|v\|_{\beta}^{p}-\Theta(u, v)=\phi(u, v) .
\end{aligned}
$$

It is easy to show that the critical points of the functional $I_{\lambda}$ and the weak solutions of the problem (1) are the same, and by Lemma 9, we prove our result. According to

$$
\|u\|_{\infty}=\max _{[0, T]}|u(t)| \text { and }\|u\|_{\infty}=\max _{[0, T]}|u(t)|
$$

taking (13) and (20) into account, one has

$$
\max _{r t \in[0, T]}|u(t)|^{p} \leq M\|u\|_{\alpha}^{p} \text { and } \max _{t \in[0, T]}|v(t)|^{p} \leq M\|v\|_{\beta}^{p}
$$

for every $(u, v) \in X$.

Hence,

$$
\max _{t \in[0, T]}\left(|u(t)|^{p}+|v(t)|^{p}\right) \leq M\left(\|u\|_{\alpha}^{p}+\|v\|_{\beta}^{p}\right)
$$

So, for every $r>0$, from the definition of and by using (61), one has

$$
\begin{aligned}
\left.\left.\phi^{-1}(]-\infty, r\right]\right):= & \{(u, v) \in X: \phi(u, v) \leq r\} \\
\subseteq & \left\{(u, v) \in X: \frac{1}{p}\|u\|_{\alpha}^{p}+\frac{1}{p}\|v\|_{\beta}^{p} \leq \frac{r}{k}\right\} \\
\subseteq & \left\{(u, v) \in X: \frac{(\Gamma(\alpha))^{p}((\alpha-1) q+1)^{p / q}}{T^{p \alpha-1}}\|u\|_{\infty}^{p}\right. \\
& \left.+\frac{(\Gamma(\beta))^{p}((\beta-1) q+1)^{p / q}}{T^{p \beta-1}}\|u\|_{\infty}^{p} \leq \frac{r}{k}\right\} \\
\subseteq & \left\{(u, v) \in X: \frac{1}{p}|u|^{p}+\frac{1}{p}|v|^{p} \leq \frac{M r}{k}, \text { for all } t \in[0, T]\right\} .
\end{aligned}
$$

Set

$$
\varphi(r)=\inf _{\left.\left.(u, v) \in \phi^{-1}(]-\infty, r\right]\right)} \frac{\sup _{\left.\left.(x, y) \in \phi^{-1}(]-\infty, r\right]\right)} \Psi(x, y)-\Psi(u, v)}{r-\phi(u, v)}
$$

Note that $\phi(0,0)=0$, and from the condition $(H 1), \Psi(0$ $, 0) \geq 0$. Hence, for every $r>0$,

$$
\begin{aligned}
\varphi(r) & =\inf _{\left.\left.(u, v) \in \phi^{-1}(]-\infty, r\right]\right)} \frac{\left(\sup _{\left.\left.(x, y) \in \phi^{-1}(]-\infty, r\right]\right)} \Psi(x, y)\right)-\Psi(u, v)}{r-\phi(u, v)} \\
& \leq \frac{\sup _{\left.\left.(x, y) \in \phi^{-1}(]-\infty, r\right]\right)} \Psi(x, y)}{r},
\end{aligned}
$$

and it follows from (68) that

$$
\varphi(r) \leq \frac{1}{r} \sup _{\Omega(M r / k)} \int_{0}^{T} F(t, u, v) d t
$$

where

$$
\Omega\left(\frac{M r}{k}\right)=\left\{(u, v) \in X: \frac{1}{p}|u(t)|^{p}+\frac{1}{p}|v(t)|^{p} \leq \frac{M r}{k}, \forall t \in[0, T]\right\} .
$$


Let $\left\{\xi_{n}\right\}$ be a sequence of positive numbers such that $\xi_{n} \longrightarrow+\infty$ and

$\lim _{\xi \rightarrow+\infty} \inf \frac{\int_{0}^{T} \sup _{|x|+|y| \leq \xi} F(t, x, y) d t}{\xi^{p}}=A_{\infty}<+\infty$.

Put $r_{n}=\left(k / p 2^{p} M\right) \xi_{n}^{p}$ for all $n \in \mathbb{N}$. Let $(u, v) \in \phi^{1}(] 1-\infty$, $\left.\left.r_{n}\right]\right)$, by (68) one has

$$
\frac{1}{p}|u(t)|^{p}+\frac{1}{p}|v(t)|^{p} \leq \frac{M}{k} r_{n}, \quad \forall t \in[0, T],
$$

which implies

$$
|u(t)| \leq \sqrt[p]{\frac{p M}{k} r_{n}} \text { and }|v(t)| \leq \sqrt[p]{\frac{p M}{k} r_{n}} .
$$

Hence, for $n$ large enough $\left(r_{n}>1\right)$

$$
|u(t)|+|v(t)| \leq 2 \sqrt[p]{\frac{p M}{k} r_{n}}=\xi_{n} .
$$

Thus, for all $n \in \mathbb{N}$,

$$
\begin{aligned}
\varphi\left(r_{n}\right) & =\frac{p 2^{p} M}{k \xi_{n}^{p}} \sup _{\left\{(u, v) \in X:|u(t)|+|v(t)|<\xi_{n}, \forall t \in[0, T]\right\}} \int_{0}^{T} F(t, u(t), v(t)) d t \\
& \leq \frac{p 2^{p} M}{k} \cdot \frac{\int_{0}^{T} \sup _{|x|+|y|<\xi_{n}} F(t, x, y) d t}{\xi_{n}^{p}} .
\end{aligned}
$$

Let

$$
\gamma:=\lim _{r \rightarrow+\infty} \inf \varphi(r) .
$$

Then,

$$
\begin{aligned}
\gamma & \leq \lim _{n \rightarrow+\infty} \inf \varphi\left(r_{n}\right) \leq \frac{p 2^{p} M}{k} \cdot \lim _{n \rightarrow+\infty} \frac{\int_{0}^{T} \sup _{|x|+|y|<\xi_{n}} F(t, x, y) d t}{\xi_{n}^{p}} \\
& =\frac{p 2^{p} M}{k} A_{\infty}<+\infty .
\end{aligned}
$$

Hence, $\Lambda \sqsubseteq] 0,1 / \gamma[$.

For $\lambda \in \Lambda$, we shall show that the functional $I_{\lambda}$ is unbounded from below.

Indeed, since $B_{\infty} / \rho^{\Delta}>1 / \lambda$, we can choose a sequence $\left\{\eta_{n}\right\}$ of positive numbers and $\varepsilon>0$ such that $\eta_{n} \longrightarrow+\infty$ and

$$
\frac{1}{\lambda}<\varepsilon<\frac{1}{\rho^{\Delta}} \cdot \frac{\int_{\theta T}^{(1-\theta) T} F\left(t, \Gamma(2-\alpha) \eta_{n}, \Gamma(2-\beta) \eta_{n}\right) d t}{\eta_{n}^{p}},
$$

For all $n \in \mathbb{N}$, and $(0,1 / p)$ define $\omega_{n}(t)=\left(\omega_{1, n}(t), \omega_{2, n}(t)\right)$ by setting

$$
\begin{gathered}
\omega_{1, n}(t)= \begin{cases}\frac{\Gamma(2-\alpha)}{\theta T} t, & t \in[0, \theta T[, \\
\Gamma(2-\alpha) \eta_{n}, & t \in[\theta T,(1-\theta) T], \\
\frac{\Gamma(2-\alpha) \eta_{n}}{\theta T}(T-t), & t \in](1-\theta) T, T],\end{cases} \\
\omega_{2, n}(t)= \begin{cases}\frac{\Gamma(2-\beta) \eta_{n}}{\theta T} t, & t \in[0, \theta T[ \\
\Gamma(2-\alpha) \beta \eta_{n}, & t \in[\theta T,(1-\theta) T], \\
\frac{\Gamma(2-\beta) \eta_{n}}{\theta T}(T-t), & t \in](1-\theta) T, T] .\end{cases}
\end{gathered}
$$

Clearly $\omega_{i, n}(0)=\omega_{i, n}(T)=0$ and $\omega_{i, n} \in L^{p}([0, T])$ for $i=1,2$. A direct calculation shows that

$$
{ }_{0} D_{t}^{\alpha} \omega_{1, n}(t)= \begin{cases}\frac{\eta_{n}}{\theta T} t^{1-\alpha}, & t \in[0, \theta T[, \\ \frac{\eta_{n}}{\theta T}\left(t^{1-\alpha}-(t-\theta T)^{1-\alpha}\right), & t \in[\theta T,(1-\theta) T], \\ \frac{\eta_{n}}{\theta T}\left(t^{1-\alpha}-(t-\theta T)^{1-\alpha}-(t-(1-\theta) T)^{1-\alpha}\right), & t \in](1-\theta) T, T],\end{cases}
$$

$$
{ }_{0} D_{t}^{\beta} \omega_{2, n}(t)= \begin{cases}\frac{\eta_{n}}{\theta T} t^{1-\beta}, & t \in[0, \theta T[, \\ \frac{\eta_{n}}{\theta T}\left(t^{1-\beta}-(t-\theta T)^{1-\beta}\right), & t \in[\theta T,(1-\theta) T], \\ \frac{\eta_{n}}{\theta T}\left(t^{1-\beta}-(t-\theta T)^{1-\beta}-(t-(1-\theta) T)^{1-\beta}\right), & t \in](1-\theta) T, T] .\end{cases}
$$

Furthermore,

$$
\begin{aligned}
\int_{0}^{T}\left|{ }_{0} D_{t}^{\alpha} \omega_{1, n}(t)\right|^{p} d t=\int_{0}^{\theta T}+\int_{\theta T}^{(1-\theta) T}+\int_{(1-\theta) T}^{T}\left|{ }_{0} D_{t}^{\alpha} \omega_{1, n}(t)\right|^{p} d t \\
=\frac{\eta_{n}^{P}}{(\theta T)^{p}}\left\{\int_{0}^{\theta T} t^{p(1-\alpha)} d t+\int_{\theta T}^{(1-\theta) T}\left(t^{1-\alpha}-(t-\theta T)^{1-\alpha}\right)^{p} d t\right. \\
\left.\quad+\int_{(1-\theta) T}^{T}\left[\left(t^{1-\alpha}-(t-\theta T)^{1-\alpha}\right)-\left(t-((1-\theta) T)^{1-\alpha}\right)\right]^{p}\right\} \\
=p P(\alpha, \theta) \eta_{n}^{p},
\end{aligned}
$$

$$
\begin{aligned}
\int_{0}^{T}\left|{ }_{0} D_{t}^{\beta} \omega_{2, n}(t)\right|^{p} d t=\int_{0}^{\theta T}+\int_{\theta T}^{(1-\theta) T}+\int_{(1-\theta) T}^{T}\left|{ }_{0} D_{t}^{\beta} \omega_{1, n}(t)\right|^{p} d t \\
=\frac{\eta_{n}^{P}}{(\theta T)^{p}}\left\{\int_{0}^{\theta T} t^{p(1-\beta)} d t+\int_{\theta T}^{(1-\theta) T}\left(t^{1-\beta}-(t-\theta T)^{1-\beta}\right)^{p} d t\right. \\
\left.\quad+\int_{(1-\theta) T}^{T}\left[\left(t^{1-\beta}-(t-\theta T)^{1-\beta}\right)-\left(t-((1-\theta) T)^{1-\beta}\right)\right]^{p}\right\} \\
=p Q(\beta, \theta) \eta_{n}^{p} .
\end{aligned}
$$

for $n$ large enough. 
Thus, $\omega_{n} \in X$, and

$$
\begin{aligned}
& \left\|\omega_{1, n}(t)\right\|^{p}=\int_{0}^{T}\left|{ }_{0} D_{t}^{\alpha} \omega_{1, n}(t)\right|^{p} d t=p P(\alpha, \theta) \eta_{n}^{p}, \\
& \left\|\omega_{2, n}(t)\right\|^{p}=\int_{0}^{T}\left|{ }_{0} D_{t}^{\alpha} \omega_{1, n}(t)\right|^{p} d t=p Q(\beta, \theta) \eta_{n}^{p} .
\end{aligned}
$$

This and (61) imply that

$$
\begin{aligned}
\Phi\left(\omega_{1, n}, \omega_{2, n}\right) & =\frac{1}{p}\left\|\omega_{1, n}(t)\right\|^{p}+\frac{1}{p}\left\|\omega_{2, n}(t)\right\|^{p}-\Theta\left(\omega_{1, n}, \omega_{2, n}\right) \\
& \leq \frac{\rho}{p}\left(\left\|\omega_{1, n}(t)\right\|^{p}+\left\|\omega_{2, n}(t)\right\|^{p}\right) \\
& =\rho(P(\alpha, \theta)+Q(\beta, \theta)) \eta_{n}^{p} \leq \rho \Delta \eta_{n}^{p} .
\end{aligned}
$$

From (H2), we have

$$
\begin{aligned}
\Psi\left(\omega_{1, n}, \omega_{2, n}\right) & =\int_{0}^{\theta T}+\int_{\theta T}^{(1-\theta)^{T}}+\int_{(1-\theta)^{T}}^{T} F\left(t, \omega_{1, n}, \omega_{2, n}\right) d t \\
& \geq \int_{\theta T}^{(1-\theta)^{T}} F\left(t, \omega_{1, n}, \omega_{2, n}\right) d t \\
& =\int_{\theta T}^{(1-\theta)^{T}} F\left(t, \Gamma(2-\alpha) \eta_{n}, \Gamma(2-\beta) \eta_{n}\right) d t .
\end{aligned}
$$

According to (80), (88), and (89), we have

$$
\begin{aligned}
I_{\lambda}\left(\omega_{1, n}, \omega_{2, n}\right)= & \phi\left(\omega_{1, n}, \omega_{2, n}\right)-\lambda \Psi\left(\omega_{1, n}, \omega_{2, n}\right) \\
\leq & \rho(P(\alpha, \theta)+Q(\beta, \theta)) \eta_{n}^{p} \\
& -\lambda \int_{\theta T}^{(1-\theta) T} F\left(t, \Gamma(2-\alpha) \eta_{n}, \Gamma(2-\beta) \eta_{n}\right) d t \\
\leq & \rho \Delta(1-\lambda \varepsilon) \eta_{n}^{p},
\end{aligned}
$$

for $n$ large enough. Taking into account the choice of $\varepsilon$, the above inequality shows that

$$
\lim _{n \rightarrow+\infty} I_{\lambda}\left(\omega_{1, n}, \omega_{1, n}\right)=-\infty \text {, }
$$

which implies that the functional $I_{\lambda}$ is unbounded from below and the claim follows.

By using the case (1) of Lemma 9, the functional $I_{\lambda}$ has a sequence $\left\{\left(u_{n}, v_{n}\right)\right\}$ of critical points such that

$$
\Phi\left(u_{n}, v_{n}\right) \longrightarrow+\infty
$$

From (22) and (61), we get

$$
\left\|\left(u_{n}, v_{n}\right)\right\|_{X} \geq p \sqrt{\frac{p \Phi\left(u_{n}, v_{n}\right)}{\rho}},
$$

which implies $\left\|\left(u_{n}, v_{n}\right)\right\|_{X} \longrightarrow+\infty$ and the proof of Theorem 10 is complete.

Theorem 11. Assume that $k>0$ and (HO) and (H2) hold. Furthermore, (H4) $F(t, 0,0)=0$ for all $t \in[0, T]$.

(H5) There exists $\theta \in(0,1 / p)$ such that, if we put

$$
\begin{aligned}
& A_{0}=\lim _{\xi \rightarrow 0^{+}} \inf \frac{\int_{0}^{T} \sup _{|x|+|y| \leq \xi} F(t, x, y) d t}{\xi^{p}}, \\
& B_{0}=\lim _{\xi \rightarrow 0^{+}} \sup \frac{\int_{\theta T}^{(1-\theta) T} F(t, \Gamma(2-\alpha) \xi, \Gamma(2-\beta) \xi) d t}{\xi^{p}},
\end{aligned}
$$

one has

$$
A_{0}<\frac{k}{2 p M \rho \Delta} B_{0}
$$

where $\Delta=\max \{P(\alpha, \theta), Q(\beta, \theta)\}$ and $M$ is given in (45).

Then, for every

$$
\left.\lambda \in \Lambda^{\prime}:=\right] \frac{\rho \Delta}{B_{0}}, \frac{k}{2 p M A_{0}}[
$$

(1) has a sequence $\left\{\left(u_{n}, v_{n}\right)\right\}$ of weak solutions such that $\left(u_{n}, v_{n}\right) \rightarrow(0,0)$.

Proof. Our goal is to apply part (2) of Lemma 9 to $\phi$ and $\Psi$ defined in (48) and (51), respectively.

As it has been pointed out before, the functionals $\phi$ and $\Psi$ satisfy the assumption regularity required in Lemma 9.

Since $F(t, 0,0)=0$ for all $t \in[0, T]$, then

$$
\min _{(u, v) \in X} \phi(u, v)=\phi(0,0)=0
$$

Let $\left\{\xi_{n}\right\}$ be a sequence of positive numbers such that $\xi_{n} \longrightarrow 0$ and

$$
\lim _{n \rightarrow+\infty} \frac{\int_{0}^{T} \sup _{|x|+|y| \leq \xi_{n}} F(t, x, y) d t}{\xi_{n}^{p}}=A_{0}<+\infty .
$$

Setting $r_{n}=\left(k / p 2^{p} M\right) \xi_{n}^{P}$ for all $n \in \mathbb{N}$, and working as in the proof of Theorem 10, we can show that

$$
\begin{aligned}
\delta & =\lim _{r \rightarrow\left(\inf _{X} \Phi\right)} \inf \varphi(r) \leq \frac{p 2^{p} M}{k} \cdot \lim _{n \rightarrow+\infty} \frac{\int_{0}^{T} \sup _{|x|+|y| \leq \xi_{n}} F(t, x, y) d t}{\xi_{n}^{p}} \\
& =\frac{p 2^{p} M}{k} A_{0},
\end{aligned}
$$

and so $\Lambda^{\prime} \subset(0,1 / \delta)$. 
Now fix $\lambda$ as in the conclusion, then

$$
\frac{1}{\lambda}<\frac{1}{\rho \Delta} \lim _{\xi \rightarrow 0^{+}} \sup \frac{\int_{\theta T}^{(1-\theta) T} F(t, \Gamma(2-\alpha) \xi, \Gamma(2-\beta) \xi) d t}{\xi^{p}},
$$

and there exist a sequence $\left\{\tau_{n}\right\}$ of positive numbers and a constant $\varepsilon_{1}$ such that $\tau_{n} \leq 1 / n$ and

$$
\begin{aligned}
& \lim _{n \rightarrow+\infty} \frac{\int_{\theta T}^{(1-\theta) T} F\left(t, \Gamma(2-\alpha) \tau_{n}, \Gamma(2-\beta) \tau_{n}\right) d t}{\tau_{n}^{p}} \\
& \quad=\lim _{\xi \rightarrow 0^{+}} \sup \frac{\int_{\theta T}^{(1-\theta) T} F(t, \Gamma(2-\alpha) \xi, \Gamma(2-\beta) \xi) d t}{\xi^{p}},
\end{aligned}
$$

and in addition

$$
\frac{1}{\lambda}<\varepsilon_{1}<\frac{1}{\rho \Delta} \lim _{n \rightarrow+\infty} \frac{\int_{\theta T}^{(1-\theta) T} F\left(t, \Gamma(2-\alpha) \tau_{n}, \Gamma(2-\beta) \tau_{n}\right) d t}{\tau_{n}^{p}} .
$$

For all $n \in \mathbb{N}$, and $\theta \in(0,1 / p)$ define $\omega_{n}(t)=\left(\omega_{1, n}(t)\right.$, $\left.\omega_{2, n}(t)\right)$ by setting

$$
\begin{gathered}
\omega_{1, n}(t)= \begin{cases}\frac{\Gamma(2-\alpha) \tau_{n}}{\theta T} t, & t \in[0, \theta T[, \\
\Gamma(2-\alpha) \tau_{n}, & t \in[\theta T,(1-\theta) T], \\
\frac{\Gamma(2-\alpha) \tau_{n}}{\theta T}(T-t), & t \in](1-\theta) T, T],\end{cases} \\
\omega_{2, n}(t)= \begin{cases}\frac{\Gamma(2-\beta) \tau_{n}}{\theta T} t, & t \in[0, \theta T[, \\
\Gamma(2-\beta) \tau_{n}, & t \in[\theta T,(1-\theta) T], \\
\frac{\Gamma(2-\beta) \tau_{n}}{\theta T}(T-t), & t \in](1-\theta) T, T] .\end{cases}
\end{gathered}
$$

Clearly $\omega_{i, n}(0)=\omega_{i, n}(T)=0$ for $i=1,2$, and $\left\{\omega_{n}\right\}$ converges strongly to $(0,0)$ in $X$.

By the same argument as in Theorem 10, we have

$$
\begin{aligned}
& I_{\lambda}\left(\omega_{1, n}, \omega_{2, n}\right)-\lambda \Psi\left(\omega_{1, n}, \omega_{2, n}\right) \leq \rho(P(\alpha, \theta)+Q(\beta, \theta)) \tau_{n}^{p} \\
& \quad-\lambda \int_{\theta T}^{(1-\theta) T} F\left(t, \Gamma(2-\alpha) \tau_{n}, \Gamma(2-\beta)_{\tau_{n}}\right) d t \\
& \leq \rho \Delta\left(1-\lambda \varepsilon_{1}\right) \tau_{n}^{p}<0=I_{\lambda}(0,0),
\end{aligned}
$$

for $n$ large enough. This together with the fact that $\left\|\omega_{n}\right\|_{X}$ $=\left\|\omega_{1, n}, \omega_{2, n}\right\|_{X} \longrightarrow 0$ shows that $I_{\lambda}$ has no local minimum at zero, and the claim follows.

The alternative of Lemma 9 case (2) ensures the existence of sequence $\left\{\left(u_{n}, v_{n}\right)\right\}$ of pairwise distinct local minima of $I_{\lambda}$ which weakly converges to $(0,0)$. This completes the proof of Theorem 11.
Finally, we present an example to illustrate our main results.

Example 12. Consider the following fractional differential system:

$\begin{cases}{ }_{t} D_{1}^{0,6}\left(\Phi_{3}\left({ }_{0} D_{t}^{0,6} u(t)\right)\right)=\lambda F_{u}(t, u(t), v(t))+\left(\sin \left(\frac{u_{1}}{2}\right)\right)^{2}, & \text { a.e.t } \in[0, T], \\ { }_{t} D_{1}^{0,75}\left(\Phi_{3}\left({ }_{0} D_{t}^{0,75} u(t)\right)\right)=\lambda F_{v}(t, u(t), v(t))+\left(\arctan \left(\frac{u_{2}}{3}\right)\right)^{2}, & \text { a.e.t } \in[0, T], \\ u(0)=u(1)=0, & v(0)=v(1)=0,\end{cases}$

where $T=1, \alpha=0,6, \beta=0,75$, and $h_{1}\left(u_{1}\right)=\left(\sin \left(u_{1} / 2\right)\right)^{2}, h_{2}$ $\left(u_{2}\right)=\left(\arctan \left(u_{2} / 3\right)\right)^{2}$. Moreover, for all $(t, u, v) \in[0,1] \times$ $\mathbb{R}^{2}$ put

$$
F(t, u(t), v(t))=\left(1+t^{2}\right) H(u, v)
$$

where

$H(u, v)= \begin{cases}\xi_{n+1}^{3} \exp \left(\frac{-1}{1-\left(u-0.8873 \xi_{n+1}\right)^{2}+\left(v-0.9064 \xi_{n+1}\right)^{2}}\right), & (u, v) \in \Omega, \\ 0, & (u, v) \in \mathbb{R}^{2} \backslash \Omega,\end{cases}$

where

$$
\Omega=\cup_{n \geq 1}\left\{(u, v):\left(u-0.8873 \xi_{n+1}\right)^{2}+\left(v-0.9064 \xi_{n+1}\right)<1\right\},
$$

and $\xi_{1}=1, \xi_{n+1}=n\left(\xi_{n}\right)^{4 / 3}+1$ for all $n \in \mathbb{N}$.

Clearly, $h_{1}, h_{2}: \mathbb{R} \longrightarrow \mathbb{R}$ are two Lipschitz continuous functions of order 2 with Lipschitzian constants $L_{1}=1 / 2$, $L_{2}=1 / 3$ and $h_{1}(0)=h_{2}(0)=0, F(t, 0,0)=0$ for all $t \in[0,1]$. With the aid of direct computation we have that

$$
M \approx 1.8925, k \approx 0.2991, \rho \approx 1.7009 .
$$

Let $\theta=1 / 3$, then we have

$$
\begin{aligned}
P\left(\alpha, \frac{1}{3}\right) & =P\left(0,6, \frac{1}{3}\right) \approx 0.3366, Q\left(\beta, \frac{1}{3}\right)=Q\left(0,75, \frac{1}{3}\right) \\
& \approx 0.3745 .
\end{aligned}
$$

Then, $\Delta \approx 0.3745$. Thus, all conditions of Theorem 10 are satisfied.

In fact, the conditions $(H 0),(H 1)$, and $(H 2)$ hold. For all $n \in \mathbb{N}$.

Restriction of $H(u, v)$ on $\Omega$ attains its maximum in $\left(0.8873_{\xi_{n+1}}, 0.9064_{\xi_{n+1}}\right)$ and

$$
H\left(0.8873_{\xi n+1}, 0.9064_{\xi_{n+1}}\right)=\xi_{n+1}^{3} \exp (-1) .
$$


In addition,

$$
\sup _{|u|+|v| \leq 0,8873 \xi_{n+1}^{-1}} H(u, v)=\xi_{n}^{3} \exp (-1),
$$

and so

$$
\begin{aligned}
B_{\infty} & =\lim _{n \rightarrow+\infty} \sup _{n \rightarrow 3} \frac{\int_{1 / 3}^{2 / 3} H\left(0.8873 \xi_{n+1}, 0.9064 \xi_{n+1}\right) d t}{\xi_{n}^{2}+1} \\
& =\lim _{n \rightarrow+\infty} \frac{\xi_{n+1}^{3} \exp (-1)}{\xi_{n}^{2}+1}=+\infty, \\
A_{\infty} & =\lim _{n \rightarrow+\infty} \inf \frac{\int_{0}^{1} \sup _{|u|+|v| \leq\left(0.8873 \xi_{n+1}\right)-1} H(u, v) d t}{\left(0.8873 \xi_{n+1}\right)^{2}} \\
& =\lim _{n \rightarrow+\infty} \frac{\xi_{n}^{3} \exp (-1)}{\left(0.8873 \xi_{n+1}\right)^{2}}=0<\frac{k}{2 p M \rho \Delta} B_{0},
\end{aligned}
$$

which implies that the condition (H3) holds. Hence, owing to Theorem 10 , for each $\lambda \in(0 ;+\infty)$, the coupled system (107) has an unbounded sequence of weak solutions.

\section{Data Availability}

No data were used to support this study.

\section{Conflicts of Interest}

The authors declare that they have no conflicts of interest.

\section{Authors' Contributions}

All authors contributed equally and significantly in writing this article. All authors read and approved the final manuscript.

\section{References}

[1] R. Hilfer, Applications of fractional calculus in physics, World Scientific, Singapore, 2000.

[2] R. Aounallah, S. Boulaaras, A. Zarai, and B. Cherif, "General decay and blow up of solution for a nonlinear wave equation with a fractional boundary damping," Mathematical Methods in the Applied Sciences, vol. 43, no. 12, pp. 7175-7193, 2020.

[3] F. Jiao and Y. Zhou, "Existence results for fractional boundary value problem via critical point theory," International Journal of Bifurcation and Chaos in Applied Sciences and Engineering, vol. 22, no. 4, p. 1250086, 2012.

[4] A. A. Kilbas, H. M. Srivastava, and J. J. Trujillo, Theory and applications of fractional differential equations, Elsevier, Amsterdam, Netherlands, 2006.

[5] V. Kokilashvili, A. Meskhi, and M. A. Ragusa, "Weighted extrapolation in grand Morrey spaces and applications to partial differential equations," Rendiconti Lincei - Matematica e Applicazioni, vol. 30, no. 1, pp. 67-92, 2019.

[6] V. Lakshmikantham and A. S. Vatsala, "Basic theory of fractional di erential equations," Nonlinear Analysis, vol. 6, pp. 2677-2682, 2008.
[7] J. Mawhin and M. Willem, Critical Point Theory and Hamiltonian Systems, Springer, New York, NY, USA, 1989.

[8] G. A. Afrouzi, N. T. Chung, and A. Hadjian, "Three solutions for a class of Neumann doubly eigenvalue boundary value systems driven by a $\left(p_{1}, \cdots, p_{n}\right)$ Laplacian operator," Le Matematiche, vol. 67, pp. 43-55, 2012.

[9] A. Singh and P. K. Banerji, "Cauchy representation of fractional Fourier transform for Boehmians," Boletim da Sociedade Paranaense de Matemática, vol. 38, no. 1, pp. 55-65, 2020.

[10] C. Bai, "Impulsive periodic boundary value problems for fractional differential equation involving Riemann-Liouville sequential fractional derivative," Journal of Mathematical Analysis and Applications, vol. 384, no. 2, pp. 211-231, 2011.

[11] K. Parand and M. Delkhosh, "Systems of nonlinear Volterra integro-differential equations of arbitrary order," Boletim da Sociedade Paranaense de Matemática, vol. 36, no. 4, pp. 3354, 2018.

[12] Z. Bai and H. Lu, "Positive solutions for boundary value problem of nonlinear fractional differential equation," Journal of Mathematical Analysis and Applications, vol. 311, no. 2, pp. 495-505, 2005.

[13] M. Bagyalakshmi and G. SaiSundarakrishnan, "Tarig projected differential transform method to solve fractional nonlinear partial differential equations," Boletim da Sociedade Paranaense de Matemática, vol. 38, no. 3, pp. 23-46, 2019.

[14] A. H. R. Sheikhani and M. Mashoof, "Numerical solution of fractional differential equation by wavelets and hybrid functions," Boletim da Sociedade Paranaense de Matemática, vol. 36, no. 2, p. 231, 2018.

[15] G. Bonanno and G. D'Aguì, "Multiplicity results for a perturbed elliptic Neumann problem," Abstract and Applied Analysis, vol. 2010, Article ID 564363, 10 pages, 2010.

[16] P. Candito and G. D'Aguì, "Three solutions to a perturbed nonlinear discrete Dirichlet problem," Journal of Mathematical Analysis and Applications, vol. 375, no. 2, pp. 594-601, 2011.

[17] S. Heidarkhani and J. Henderson, "Multiple solutions for a nonlocal perturbed elliptic problem of p-Kirchho/ type," Communications on Applied Nonlinear Analysis, vol. 19, pp. 25-39, 2012.

[18] M. Tomar, P. Agarwal, and J. Choi, "Hermite-Hadamard type inequalities for generalized convex functions on fractal sets style," Boletim da Sociedade Paranaense de Matemática, vol. 38, no. 1, pp. 101-116, 2020.

[19] K. S. Miller and B. Ross, An Introduction to the Fractional Calculus and Fractional Differential Equations, Wiley, New York, NY, USA, 1993.

[20] I. Podlubny, Fractional Differential Equations, Academic Press, San Diego, CA, USA, 1999.

[21] P. H. Rabinowitz, "Minimax methods in critical point theory with applications to differential equations," in CBMS Regional Conference Series in Mathematics, pp. 1-110, Madison, WI, USA, 1986.

[22] B. Ricceri, "A three critical points theorem revisited," Nonlinear Analysis, vol. 70, no. 9, pp. 3084-3089, 2009.

[23] S. G. Samko, A. A. Kilbas, and O. I. Marichev, Fractional Integral and Derivatives: Theory and Applications, Gordon and Breach, Longhorne, PA, USA, 1993.

[24] J. Wang and Y. Zhou, "A class of fractional evolution equations and optimal controls," Nonlinear Analysis: Real World Applications, vol. 12, no. 1, pp. 262-272, 2011. 
[25] Z. Wei, W. Dong, and J. Che, "Periodic boundary value problems for fractional differential equations involving a RiemannLiouville fractional derivative," Nonlinear Analysis: Theory, Methods \& Applications, vol. 73, no. 10, pp. 3232-3238, 2010.

[26] F. Kamache, R. Guefaifia, S. Boulaaras, and A. Alharbi, "Existence of weak solutions for a new class of fractional p-Laplacian boundary value systems," Mathematics, vol. 8, no. 4, p. 475, 2020.

[27] S. Zhang, "Positive solutions to singular boundary value problem for nonlinear fractional differential equation," Computers \& Mathematcs with Applications, vol. 59, no. 3, pp. 13001309, 2010.

[28] S. Boulaaras, R. Guefaifia, A. Alharbi, and B. Cherif, "Cherif: existence of 3-weak solutions for a new class of an overdetermined system of fractional partial integro-differential equations," Fractal, vol. 28, no. 8, article 2040036, 9 pages, 2020.

[29] R. P. Agarwal, S. Gala, and M. A. Ragusa, "A regularity criterion in weak spaces to Boussinesq equations," Mathematics, vol. 8, no. 6, p. 920, 2020.

[30] S. Gala and M. A. Ragusa, "Logarithmically improved regularity criterion for the Boussinesq equations in Besov spaces with negative indices," Applicable Analysis, vol. 95, no. 6, pp. 12711279, 2016.

[31] D. Li, F. Chen, and Y. An, "Existence of solutions for fractional differential equation with $p$ Laplacian through variational method," Journal of Applied Analysis \& Computation, vol. 8, p. 1795, 2018.

[32] F. Kamache, R. Guefaifia, and S. Boulaaras, "Existence of three solutions for perturbed nonlinear fractional p-Laplacian boundary value systems with two control parameters," Journal of Pseudo-Differential Operators and Applications, vol. 11, no. 4, pp. 1781-1803, 2020.

[33] D. Li, F. Chen, and Y. An, "Existence and multiplicity of nontrivial solutions for nonlinear fractional differential systems with p-Laplacian via critical point theory," Mathematical Methods in the Applied Sciences, vol. 41, no. 8, pp. 31973212, 2018.

[34] S. Gala, Q. Liu, and M. A. Ragusa, "A new regularity criterion for the nematic liquid crystal flows," Applicable Analysis, vol. 91, no. 9, pp. 1741-1747, 2012.

[35] G. Bonanno and S. A. Marano, "On the structure of the critical set of non-differentiable functions with a weak compactness condition," Applicable Analysis, vol. 89, no. 1, pp. 1-10, 2010.

[36] G. Bonanno and G. M. Bisci, "Infinitely many solutions for a boundary value problem with discontinuous nonlinearities," Boundary Value Problems, vol. 2009, no. 1, Article ID 670675, 2009.

[37] B. Ricceri, "A general variational principle and some of its applications," Journal of Computational and Applied Mathematics, vol. 113, no. 1-2, pp. 401-410, 2000.

[38] Y. Zhao, H. Chen, and Q. Zhang, "Infinitely many solutions for fractional differential system via variational method," Journal of Applied Mathematics and Computing, vol. 50, no. 1-2, pp. 589-609, 2016. 